Rev. Int. Contam. Ambie. 37, 577-588, 2021

https://doi.org/10.20937/RICA.54004

\title{
ACTINOBACTERIAS DEGRADADORAS DE POLIPROPILENO
}

Polypropylene degrading actinobacteria

\author{
Micaela BOENEL ${ }^{1 *}$, Gernot VOBIS ${ }^{2}$ y Mariana SOLANS ${ }^{2}$
}

${ }^{1}$ Instituto Andino Patagónico de Tecnologías Biológicas y Geoambientales, Universidad Nacional del Comahue, Consejo Nacional de Investigaciones Científicas y Técnicas, Quintral 1250, 8400 San Carlos de Bariloche, Río Negro, Argentina.

${ }^{2}$ Instituto de Investigaciones en Biodiversidad y Medioambiente, Universidad Nacional del Comahue, Consejo Nacional de Investigaciones Científicas y Técnicas, Quintral 1250, 8400 San Carlos de Bariloche, Río Negro, Argentina.

*Autora para correspondencia: micaelaboenel@gmail.com

(Recibido: abril de 2020; aceptado: noviembre de 2020)

Palabras clave: Actinomadura, degradación de polímeros, Streptomyces

\section{RESUMEN}

La acumulación de polímeros sintéticos es un gran problema de contaminación ambiental, ya que no existen métodos eficientes para su eliminación segura; sin embargo, hay evidencias sobre su degradación por actinobacterias. En este trabajo se evaluó la capacidad de degradación de actinobacterias aisladas de suelo del vertedero de Bariloche, sobre tres biopolímeros y un polímero sintético. Por un lado, se estudió la degradación de polímeros naturales (almidón, hemicelulosa, celulosa) en medio sólido, y por otro el otro se evaluó la degradación de láminas de polipropileno inoculadas con los aislamientos y cultivadas en medio líquido durante seis semanas a $37^{\circ} \mathrm{C}$. Luego se midió la pérdida de peso en las láminas y se estudió la colonización de la superficie del polipropileno mediante microscopia óptica y electrónica. Los datos fueron analizados con un ANOVA de dos vías y la prueba de Tukey. De los 35 aislamientos totales, el 88 $\%$ correspondió al género Streptomyces y el $3 \%$ a los demás géneros (Actinomadura, Pseudonocardia, Saccharomonospora y Thermoactinomyces). El $63 \%$ de los aislamientos tuvo la capacidad de degradar almidón, el $86 \%$ hemicelulosa, el $34 \%$ celulosa microcristalina (exoglucanasas) y el $88 \%$ carboximetilcelulosa (endoglucanasas). Se observó colonización de la superficie de polipropileno en todos los tratamientos. Dos de las cepas estudiadas (Streptomyces sp. MP32 y Actinomadura sp. MP5) redujeron el peso de las láminas de polipropileno $(\mathrm{p} \leq 0.05)$. Estos resultados preliminares muestran la capacidad de las actinobacterias para colonizar la superficie del polipropileno, con potencial uso en suelos o ambientes contaminados por plásticos.

Key words: Actinomadura, polymer degradation, Streptomyces

\begin{abstract}
The accumulation of synthetic polymers is a great environmental contamination issue, since there are no efficient methods to dispose them safely; however, there is evidence
\end{abstract}


about the degradation of plastics by actinobacteria. In this work we tested the degradation capacity of tree biopolymers and a synthetic polymer by actinobacteria isolated from the soil of a landfill in Bariloche. On the one hand, we studied the degradation of natural polymers (starch, hemicellulose, cellulose) in a solid medium, and on the other hand, we studied the degradation of polypropylene sheets inoculated with the isolates and cultured in liquid medium for six weeks at $37^{\circ} \mathrm{C}$. We then measured weight loss in the sheets and studied colonization of the polypropylene surface using light and electron microscopy. The data were analyzed with a two-way ANOVA and Tukey's test. Of the 35 total isolates, $88 \%$ corresponded to the Streptomyces genus and $3 \%$ to the rest of genera (Actinomadura, Pseudonocardia, Saccharomonospora and Thermoactinomyces). Of the isolates, $63 \%$ were able to degrade starch, $86 \%$ hemicellulose, $34 \%$ microcrystalline cellulose (exoglucanases), and 88\% carboxymethyl cellulose (endoglucanases). Colonization of the polypropylene surface was observed in all treatments. Two of the strains studied, Streptomyces sp. MP32 and Actinomadura sp. MP5 reduced the weight of the polypropylene sheets $(\mathrm{p} \leq 0.05)$. These preliminary results show the ability of actinobacteria to colonize the polypropylene surface, with potential use in soils or environments contaminated by synthetic polymers.

\section{INTRODUCCIÓN}

La contaminación por acumulación de polímeros sintéticos constituye un gran problema ambiental (Shimao 2001, Mor y Sivan 2008, Krueger et al. 2015, Álvarez-Zeferino et al. 2020). Debido al creciente consumo de estos materiales se producen cerca de $140 \mathrm{Mt}$ a nivel mundial (Ojha et al. 2017), concentradas principalmente en la elaboración de polietileno (PE) y polipropileno (PP). Si bien cerca del $62 \%$ de los productos plásticos se fabrican con $\mathrm{PE}$, el PP presenta el crecimiento interanual de la tasa de consumo más alto del mundo. Este último registró una producción global de 56.44 Mt en 2016, y se espera un aumento para el año 2022 a $75.72 \mathrm{mT}$ (Arráez et al. 2018). En 2018, la producción mundial de plásticos alcanzó los $360 \mathrm{Mt}$ (https://www. plasticseurope.org).

Si bien existen métodos de eliminación para los desechos plásticos mencionados, la mayoría tiene efectos secundarios para el ambiente o son costosos (Balasubramanian et al. 2010). Como consecuencia de estos métodos ineficientes, se observa un incremento de la acumulación de polímeros sintéticos en el ambiente, la cual se presenta como una amenaza tanto en ecosistemas terrestres como acuáticos (Shimao 2001, Mor y Sivan 2008, Krueger et al. 2015, Álvarez-Zeferino et al. 2020), ya que en su mayoría no pueden degradarse mediante factores extrínsecos de la naturaleza (Ojha et al. 2017). Existe consenso en que el proceso de biodegradación de polímeros sintéticos es extremadamente lento cuando ocurre sin ningún tipo de intervención (Restrepo-Flórez et al. 2014), lo cual se debe a la insolubilidad de estos materiales en medios acuosos, la falta de grupos funcionales y su alto peso molecular. Dado que las superficies de los polímeros sintéticos están diseñadas para ser lisas, hidrófobas y no adsorber productos químicos (Mason et al. 2016, Ojha et al. 2017), la degradación de estos materiales por microorganismos es limitada (Krueger et al. 2015). Sin embargo, al ser descartados y quedar expuestos a la luz solar y ultravioleta (Arráez et al. 2018, Hossain et al. 2018), así como a la congelación y descongelación, la estructura de los polímeros sintéticos sufre erosión y sus superficies se vuelven relativamente más rugosas e hidrofóbicas, lo cual favorece la colonización por microorganismos (Singh y Sharma 2008, Oberbeckmann et al. 2015, Ojha et al. 2017).

Entre los organismos que tienen la capacidad de colonizar los plásticos se encuentran las actinobacterias. Éstas constituyen un grupo morfológico y fisiológicamente heterogéneo que puede sintetizar y excretar una gran diversidad de metabolitos secundarios como enzimas, terpenoides, pigmentos y otras moléculas (Bowen y Rovira 1999, Amoroso et al. 2013, Polti et al. 2014). También pueden degradar estructuras complejas como almidón, celulosa, hemicelulosa, lignina, lignocelulosa, pectina, queratina, humus y quitina (Vobis y Chaia 1998, Amoroso et al. 2013, Polti et al. 2014). Diversos estudios han mostrado su capacidad de degradar distintos tipos de polímeros sintéticos (Mergaert y Swings 1996, Sivan et al. 2006, Mor y Sivan 2008, Sukkhum et al. 2009a). Por tal motivo, las actinobacterias resultan muy atractivas para su investigación en cuanto a la degradación de polímeros complejos como el PP. 
El objetivo de este trabajo fue estudiar la capacidad de degradación de actinobacterias aisladas del vertedero municipal de San Carlos de Bariloche, Río Negro, Argentina, sobre distintos biopolímeros (almidón, hemicelulosa y celulosa), y sobre un polímero sintético $(\mathrm{PP})$.

\section{MATERIALES Y MÉTODOS}

\section{Recolección de las muestras}

La ciudad de Bariloche se encuentra en el noroeste de la Patagonia. Está situada dentro del Parque Nacional Nahuel Huapi $\left(41^{\circ} 4^{\prime}-41^{\circ} 1^{\prime} \mathrm{S}, 71^{\circ} 6^{\prime}-71^{\circ} 1^{\prime}\right.$ 'O), en la costa sur del lago Nahuel Huapi, provincia de Río Negro, Argentina (Bühler et al. 2013).

Las muestras de sustrato (suelo) fueron tomadas del vertedero municipal de San Carlos de Bariloche, ubicado sobre el sector sudoeste, en la zona conocida como Pampa de Huenuleo, valle comprendido entre los cerros Otto, Ventana, Nireco, Carbón y la morena glaciar en la que se asientan barrios del casco urbano de la ciudad. Las muestras de sustrato se tomaron a una profundidad de $5-15 \mathrm{~cm}$ con una pala de jardín desinfectada y se colocaron en recipientes estériles (una palada por recipiente). Las muestras se recogieron por triplicado y fueron secadas a temperatura ambiente en el laboratorio.

\section{Aislamiento de actinobacterias}

Se utilizó un gramo de muestra, el cual fue colocado en un tubo de vidrio con $9 \mathrm{ml}$ de agua destilada estéril, se agitó y se realizaron diluciones sucesivas de $10^{-1}$ a $10^{-5}$. Se sembró $0.1 \mathrm{~mL}$ de cada dilución sobre la superficie del medio artificial de suelo KEHE agarizado $\left(0.101 \% \mathrm{p} / \mathrm{v} \mathrm{CaSO}_{4} \cdot 2 \mathrm{H}_{2} \mathrm{O}, 0.049 \%\right.$ $\mathrm{p} / \mathrm{v} \mathrm{Ca}\left(\mathrm{NO}_{3}\right)_{2} \cdot 4 \mathrm{H}_{2} \mathrm{O}, 0.07 \% \mathrm{p} / \mathrm{v} \mathrm{MgSO}_{4} \cdot 7 \mathrm{H}_{2} \mathrm{O}$, $0.0025 \% \mathrm{p} / \mathrm{v} \mathrm{K} \mathrm{SO}_{4}, 0.0005 \% \mathrm{p} / \mathrm{v} \mathrm{K} \mathrm{K}_{2} \mathrm{HPO}_{4}, 0.02 \%$ $\mathrm{p} / \mathrm{v} \mathrm{NaHCO}_{3}$, traza $\mathrm{FeCl}_{3}, 0.01 \% \mathrm{p} / \mathrm{v}$ extracto de levadura, $0.001 \%$ glucosa, $1.5 \% \mathrm{p} / \mathrm{v}$ agar, $5 \% \mathrm{v} / \mathrm{v}$, extracto de suelo) (Vobis 2006) contenido en placas, por triplicado. Las placas se incubaron a $37{ }^{\circ} \mathrm{C}$ en estufa durante dos semanas. Se aislaron las colonias desarrolladas, se purificaron y se conservaron a $4^{\circ} \mathrm{C}$.

\section{Caracterización y determinación taxonómica de actinobacterias}

Para la descripción y caracterización morfológica de las colonias de actinobacterias, se cultivaron los aislamientos en medio de cultivo YpSs con extracto de levadura y almidón (Emerson 1958): $0.4 \% \mathrm{p} / \mathrm{v}$ extracto de levadura, $1.5 \% \mathrm{p} / \mathrm{v}$ almidón soluble, $0.1 \%$ $\mathrm{p} / \mathrm{v} \mathrm{K} \mathrm{K}_{2} \mathrm{HPO}_{4}, 0.05 \% \mathrm{p} / \mathrm{v} \mathrm{MgSO}_{4} \cdot 7 \mathrm{H}_{2} \mathrm{O}, 2 \% \mathrm{p} / \mathrm{v}$ agar).
Las observaciones de la morfología de colonias de actinobacterias se realizaron de forma directa a través de un microscopio estereoscópico con equipo de transiluminación (ME) modelo Olympus SZH, ediante la técnica de microscopia directa de Cross (1989), sobre cultivos sembrados en medio YpSs. La observación y descripción de morfología de esporas se realizó a través de un microscopio óptico (MO) modelo Olympus BX50, sobre cultivos sembrados en medio de cultivo KEHE. En ambos casos la temperatura de incubación fue de $37^{\circ} \mathrm{C}$ y el tiempo de incubación de tres semanas.

Para la identificación genérica se utilizaron características morfológicas como desarrollo de hifas y formación de esporas (Shirling y Gottlieb 1966, Miyadoh et al. 1997, Dworkin et al. 2007, Goodfellow 2012).

\section{Producción de pigmento}

Se observó la presencia de pigmentos en las colonias que crecieron en medio sólido de cultivo YpSs. Posteriormente se clasificaron los aislamientos como positivos si producían pigmento soluble $(+)$ o negativos si no producían pigmento (-).

\section{Capacidad de crecimiento a $50^{\circ} \mathrm{C}$}

Para estudiar la capacidad de crecimiento de las actinobacterias a $50^{\circ} \mathrm{C}$, los aislamientos se cultivaron en medio YpSs sólido y se incubaron en estufa durante una semana, por duplicado. Posteriormente se registraron los aislamientos que crecieron a esa temperatura.

\section{Degradación de biopolímeros in vitro}

Para evaluar la actividad enzimática específica sobre determinados biopolímeros se utilizaron medios que contenían almidón, hemicelulosa y celulosa. Se sembraron cultivos maduros en los respectivos medios y se incubaron una semana a $37^{\circ} \mathrm{C}$. Luego se registró la presencia de halos de actividad enzimática alrededor de las colonias (reacción positiva).

\section{Almidón (amilasas)}

Para la detección de actividad amilasa se utilizó el medio de cultivo sólido YpSs con almidón como fuente de energía y carbono. Una vez transcurrido el tiempo de cultivo, se cubrió la placa con iodo al $1 \%$ provocando una reacción colorimétrica violeta en zonas con presencia de almidón (reacción negativa) y halos transparentes de degradación (reacción positiva) (Rohban et al. 2008).

\section{Hemicelulosa (xilanasas)}

Para la detección de actividad xilanasa se utilizó un medio de cultivo sólido con xilano como fuente de 
carbono (XED: $0.3 \% \mathrm{p} / \mathrm{v}$ extracto de levadura, $0.7 \%$ $\mathrm{p} / \mathrm{v}$ xilano, $2.5 \% \mathrm{p} / \mathrm{v}$ agar). Una vez transcurrido el tiempo de cultivo, se cubrió la placa con iodo al $1 \%$, provocando una reacción colorimétrica violeta en zonas con presencia de xilano (reacción negativa) o halos de actividad enzimática (reacción positiva) (Rivas et al. 2003).

\section{Celulosas (exo y endoglucanasas)}

Para la detección de actividad exoglucanasa se utilizó el medio de cultivo basal 1:0.25\% p/v NH${ }_{4} \mathrm{SO}_{4}, 0.025 \% \mathrm{p} / \mathrm{v} \mathrm{K} \mathrm{HPO}_{4}, 0.01 \% \mathrm{NaCl}, 0.0125 \%$ $\mathrm{p} / \mathrm{v} \mathrm{MgSO}_{4} \cdot 7 \mathrm{H}_{2} \mathrm{O}, 0.00025 \% \mathrm{p} / \mathrm{v} \mathrm{FeSO}_{4} \cdot 7 \mathrm{H}_{2} \mathrm{O}$, $0.00025 \% \mathrm{p} / \mathrm{v} \mathrm{MnSO}_{4} \cdot 7 \mathrm{H}_{2} \mathrm{O}, 1 \% \mathrm{p} / \mathrm{v}$, celulosa microcristalina, $0.1 \% \mathrm{p} / \mathrm{v}$ extracto de levadura, $1.2 \%$ $\mathrm{p} / \mathrm{v}$ agar conteniendo celulosa microcristalina como fuente de carbono (Percival-Zhang et al. 2006). Para la detección de actividad endoglucanasa se utilizó el medio de cultivo basal 2, con carboxi-metil-celulosa sódica como fuente de carbono $(0.2 \% \mathrm{p} / \mathrm{v} \mathrm{NaNO}$, $0.1 \% \mathrm{p} / \mathrm{v} \mathrm{K} \mathrm{K}_{2} \mathrm{HPO}_{4}, 0.05 \% \mathrm{p} / \mathrm{v} \mathrm{MgSO}_{4}, 0.5 \% \mathrm{p} / \mathrm{v}$ $\mathrm{KCl}, 0.2 \% \mathrm{p} / \mathrm{v}$ carboxi-metil-celulosa sódica, $0.02 \%$ $\mathrm{p} / \mathrm{v}$ peptona, $1.7 \% \mathrm{p} / \mathrm{v}$ agar). El revelado se realizó con iodo Gram según Kasana et al. (2008).

\section{Degradación microbiana in vitro de polipropileno (PP)}

Se seleccionaron cinco aislamientos de distintos géneros de actinobacterias para evaluar la capacidad de degradación de PP. Se utilizaron láminas de 1 $\times 1 \mathrm{~cm}$ de PP, previamente pesadas (el peso de las láminas de PP estaba en el orden de $2.5-3 \mathrm{mg}$ ) y pretratadas con luz UV a $254 \mathrm{~nm}$ durante un mes para debilitar la estructura del polímero y facilitar el ataque microbiano (Restrepo-Flórez et al. 2014, Hossain et al. 2018, Ojha et al. 2017). Se estudió la colonización de la superficie de la lámina de PP por las actinobacterias mediante microscopia óptica (MO) y microscopia electrónica de barrido (MEB), y se estableció la pérdida de peso de la lámina de PP por medición gravimétrica respecto a un testigo sin inóculo de actinobacteria, como parámetros de la potencial capacidad de degradación del material. El porcentaje de pérdida de peso se determinó utilizando la siguiente fórmula:

Pérdida de peso (\%): [(peso inicial-peso final)/ (peso inicial $] \times 100$

\section{Ensayo en medio solido}

Se colocó una lámina del PP en el centro de la placa con medio KEHE y en uno de sus vértices se sembró el aislamiento de actinobacteria (en contacto directo con el PP y con el medio de cultivo). Los tratamientos fueron seis: cada una de las actinobacterias seleccionadas (cinco) en contacto con la lámina del PP y un testigo (lámina de PP sin inóculo de actinobacteria). Se realizaron 12 réplicas para cada tratamiento. La temperatura de incubación fue de $37^{\circ} \mathrm{C}$ en estufa durante 45 días. Luego se observó la colonización de la lámina con MO y ME. Finalmente se desinfectaron las láminas con alcohol $70 \% \mathrm{v} / \mathrm{v}$ durante $15 \mathrm{~min}$, se lavaron con agua destilada, se secaron en estufa y se pesaron.

\section{Ensayo en medio líquido}

Se colocaron seis láminas de PP en matraces Erlenmeyer de $250 \mathrm{ml}$ con $100 \mathrm{ml}$ del medio de cultivo KEHE líquido. Se inocularon las actinobacterias con un anza en esterilidad y se incubaron a $37^{\circ} \mathrm{C}$ en agitador orbital (modelo SI-300R) con agitación de $125 \mathrm{rpm}$ durante 45 días. Se repitieron los mismos tratamientos realizados en el ensayo anterior (cada uno de los cinco aislamientos de actinobacterias con seis láminas de PP por Erlenmeyer [pseudoréplicas] $\mathrm{y}$ un testigo que contaba con seis láminas de PP sin inocular). Se realizaron cuatro réplicas de cada tratamiento siguiendo el mismo procedimiento que en el ensayo en medio sólido, hasta obtener el peso final de las láminas.

\section{Observación con MEB}

Se extrajo una lámina de $\mathrm{PP}$ de cada tratamiento al azar, tanto en el ensayo en medio sólido como en el ensayo en medio líquido, y se fijó en glutaraldehído al $4 \%$ para su posterior visualización en el MEB (FEI Inspect S50) del Departamento de Caracterización de Materiales, Gerencia de Investigación Aplicada (CM-GIA-GAATEN-CNEA) del Centro Atómico Bariloche.

\section{Análisis de datos}

Dado que el ensayo contó con un diseño factorial, los resultados del ensayo de degradación de PP en medio sólido se analizaron mediante la prueba no paramétrica de Kruskal Wallis, en tanto que los datos obtenidos del ensayo de degradación de PP en medio líquido fueron comparados mediante un análisis de varianza de dos vías (ANOVA) para evaluar el efecto principal de cada variable independiente (distintos géneros de actinobacterias) y su posible interacción. Posteriormente se realizó la prueba de Tukey para examinar todas las diferencias entre medias de los tratamientos, con un nivel de significancia de 0.05. Para realizar los análisis estadísticos se utilizó el paquete estadístico $\mathrm{R}$ versión 3.4.2. 


\section{RESULTADOS}

\section{Aislamientos}

Se obtuvo un total de 35 aislamientos de actinobacterias presentes en el suelo del vertedero de San Carlos de Bariloche, pertenecientes a diversos géneros, con base en sus características morfológicas y anatómicas. El $88 \%$ de los aislamientos fue identificado y clasificado dentro del género Streptomyces, mientras que el $12 \%$ restante fue identificado y clasificado dentro de los géneros Actinomadura, Pseudonocardia, Saccharomonospora y Thermoactinomyces.

El $51 \%$ de los aislamientos de actinobacterias crecieron a $50{ }^{\circ} \mathrm{C}$ y correspondieron a los géneros Saccharomonospora y Streptomyces. De los 31 aislamientos del género Streptomyces, más de la mitad (17 aislamientos) crecieron y se desarrollaron a $50{ }^{\circ} \mathrm{C}$ (Cuadro I).

\section{Degradación de polímeros naturales}

Del total de aislamientos de actinobacterias, el $86 \%$ tuvo la capacidad de degradar hemicelulosa; el $88 \%$ mostró resultados positivos para endoglucanasas, encargadas de la degradación de la CMC, mientras que el 34\% mostró resultados positivos para exoglucanasas, encargadas de la degradación de la celulosa microcristalina. Por último, el $63 \%$ de los aislamientos presentó capacidad para degradar almidón (Cuadro I).

En este trabajo, 22 de los aislamientos, correspondientes a los géneros Actinomadura, Saccharomonospora, Streptomyces y Thermoactinomyces, presentaron actividad amilolítica (Cuadro I). La actividad enzimática xilanolítica se manifestó en 30 de los aislamientos, pertenecientes a los géneros Actinomadura, Saccharomonospora y Streptomyces (Cuadro I). Más del $85 \%$ de los aislamientos de actinobacterias se mostraron implicados en la degradación de celulosa, en tanto que 32 aislamientos de los géneros Actinomadura, Pseudonocardia, Saccharomonospora, Streptomyces y Thermoactinomyces exhibieron actividad endoglucanasa. Por otro lado, 12 aislamientos de actinobacterias presentaron actividad exoglucanasa, la mayoría pertenecientes al género Streptomyces,

CUADRO I. PERFIL DE ACTIVIDAD ENZIMÁTICA IMPLICADA EN LA DEGRADACIÓN DE BIOPOLÍMEROS COMPLEJOS Y CRECIMIENTO A $50^{\circ} \mathrm{C}$ DE LOS AISLAMIENTOS DE ACTINOBACTERIAS PROCEDENTES DEL VERTEDERO MUNICIPAL DE SAN CARLOS DE BARILOCHE.

\begin{tabular}{|c|c|c|c|c|c|c|}
\hline Género & $\begin{array}{l}\text { Códigos de } \\
\text { aislamientos }\end{array}$ & Amilasa & Xilanasa & Endoglucanasa & Exoglucanasa & $\begin{array}{c}\text { Crecimiento a } \\
50^{\circ} \mathrm{C}\end{array}$ \\
\hline Streptomyces sp. & MP10, MP26, MP35 & + & + & + & + & + \\
\hline Streptomyces sp. & MP34, MP36 & + & + & + & + & - \\
\hline Streptomyces sp. & MP40 & + & + & + & + & - \\
\hline $\begin{array}{l}\text { Saccharomonospora sp./ } \\
\text { Streptomyces sp. }\end{array}$ & MP29 /MP38 & + & + & + & - & + \\
\hline Streptomyces sp. & MP7 & + & + & + & nd & + \\
\hline Streptomyces sp. & $\begin{array}{l}\text { MP1, MP3, MP14, MP17, } \\
\text { MP27, MP32 }\end{array}$ & + & + & + & - & + \\
\hline Streptomyces sp. & MP18, MP31 & + & + & + & - & - \\
\hline $\begin{array}{l}\text { Actinomadura sp. } \\
\text { /Streptomyces sp. }\end{array}$ & MP5/ MP11 & + & + & + & - & - \\
\hline Streptomyces sp. & MP24, MP25, MP30 & - & + & + & + & + \\
\hline Streptomyces sp. & MP8 & - & + & + & + & - \\
\hline Streptomyces sp. & MP9 & - & + & + & + & - \\
\hline Streptomyces sp. & MP13 & - & + & + & - & + \\
\hline Streptomyces sp. & MP33 & + & + & - & - & + \\
\hline Streptomyces sp. & MP12, MP21 & - & + & + & - & - \\
\hline Streptomyces sp. & MP4 & + & nd & + & - & - \\
\hline Pseudonocardia sp. & MP39 & - & nd & + & + & - \\
\hline Thermoactinomyces sp. & MP22 & + & nd & + & - & - \\
\hline Streptomyces sp. & MP23 & - & nd & - & - & + \\
\hline Streptomyces sp. & MP37 & nd & nd & nd & nd & - \\
\hline
\end{tabular}

$(+)$ actividad positiva, $(-)$ actividad negativa, nd: no determinado. 
aunque también Pseudonocardia exhibió esta actividad (Cuadro I). De los 35 aislamientos, el $17 \%$ presentó actividad enzimática para degradar todos los biopolímeros complejos estudiados, mientras que el 51 $\%$ exhibió capacidad para degradar tres de los cuatro biopolímeros probados. El $26 \%$ posee capacidad de degradación para dos de los cuatro biopolímeros, y sólo el $6 \%$ no tuvo la capacidad de degradar ninguno de los biopolímeros probados.

\section{Degradación de polipropileno en medio sólido}

El total de los aislamientos estudiados presentaron crecimiento de micelio y desarrollo de la colonia, tanto alrededor de la lámina de PP como sobre ésta, colonizando parcialmente la misma. Todos mostraron desarrollo de hifas y esporas sobre la superficie del PP (Fig. 1); sin embargo, no se observaron diferencias (p $\geq 0.05$ ) en el peso de las láminas de PP inoculadas, comparadas con el testigo sin inocular.

\section{Degradación de polipropileno en medio líquido}

Luego de 45 días de cultivo en medio líquido en agitación, todas las actinobacterias estudiadas crecieron y se adhirieron a la lámina del PP, colonizando su superficie.

En relación con la colonización de las láminas de PP por actinobacterias, se pudieron observar diferentes grados de colonización de la superficie y desarrollo de diversas estructuras, características de cada género. Mediante MEB, se visualizó el desarrollo de hifas y esporas de Actinomadura sp. MP5 sobre la superficie de la lámina de PP (Fig. 2a), al igual que en los casos de Streptomyces sp. MP32 (Fig. 2b). Sacharamonospora sp. MP29 (Fig. 2c), Thermoactinomyces sp. MP22 (Fig 2d) y Pseudonocardia sp. MP39 (Fig. 2e).

Respecto de la pérdida de peso, las láminas de PP incubadas con el aislamiento MP32 del género Streptomyces presentaron un valor significativamente menor con relación a los pesos de las láminas de $\mathrm{PP}$ del testigo (sin inóculo) $(\mathrm{P}=2.25$ $\times 10^{-6}$ y $\left.\alpha=0.05\right)$. De igual manera ocurrió con el aislamiento MP5 del género Actinomadura, en el cual los pesos de las láminas de PP incubadas con este aislamiento mostraron un valor significativamente menor respecto a los pesos de las láminas de $\mathrm{PP}$ del testigo ( sin actinobacteria) $(\mathrm{P}=0.0044$ y $\alpha=0.05)$. Estas pérdidas de peso representan el $25.39 \%$ para MP32 y el $12.83 \%$ para MP5. En cuanto a las cepas de Saccharomonospora sp. MP29, Thermoactinomyces sp. MP22 y Pseudonocardia sp. MP39, no se observaron diferencias respecto a los testigos $(\mathrm{p}>0.05)$ durante los 45 días de incubación (Fig. 3).

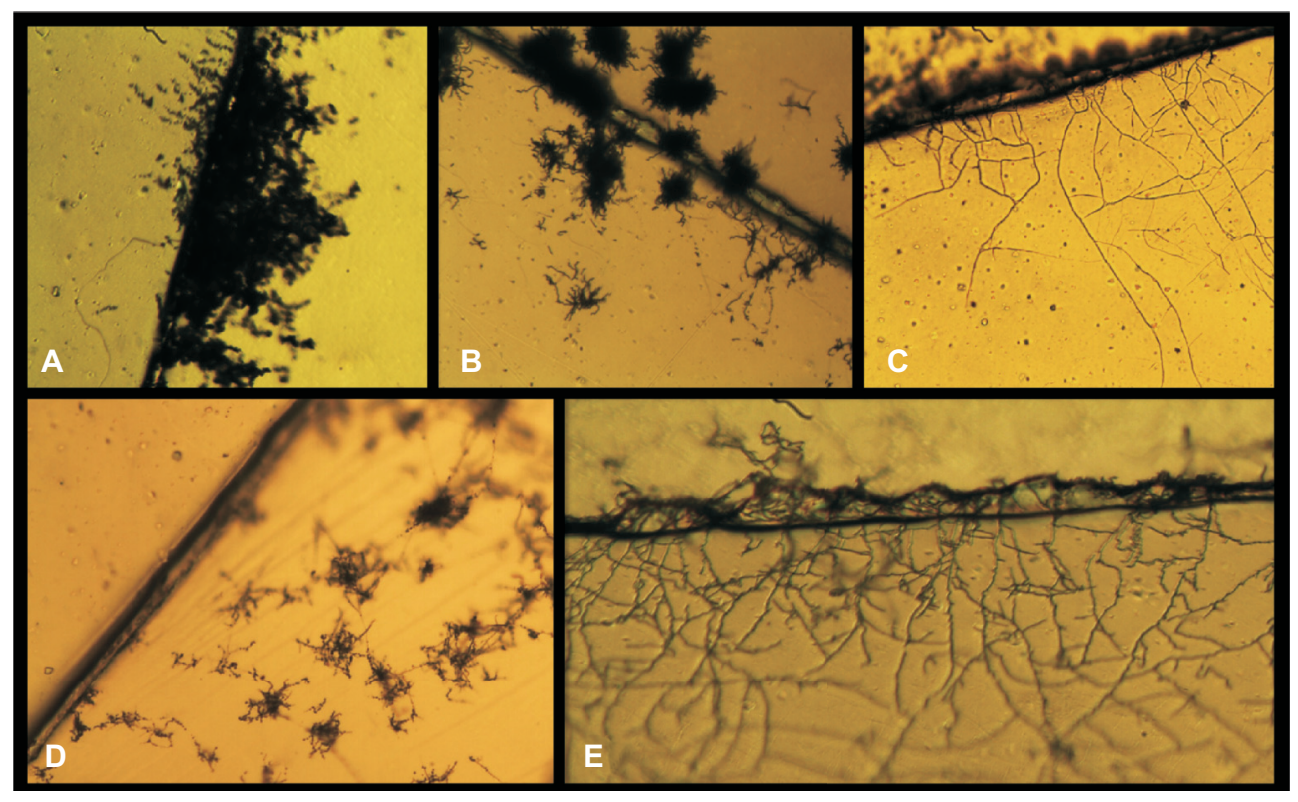

Fig. 1. Crecimiento de distintas cepas de actinobacterias en medio sólido KEHE en contacto con láminas de PP. Se observa un desarrollo del micelio más denso a lo largo de los bordes de la lámina. (a) Actinomadura sp. MP5; (b) Streptomyces sp. MP32; (c) Saccharomonospora sp MP29; (d) Thermoactinomyces sp. MP22, y (e) Pseudonocardia sp. MP39. 


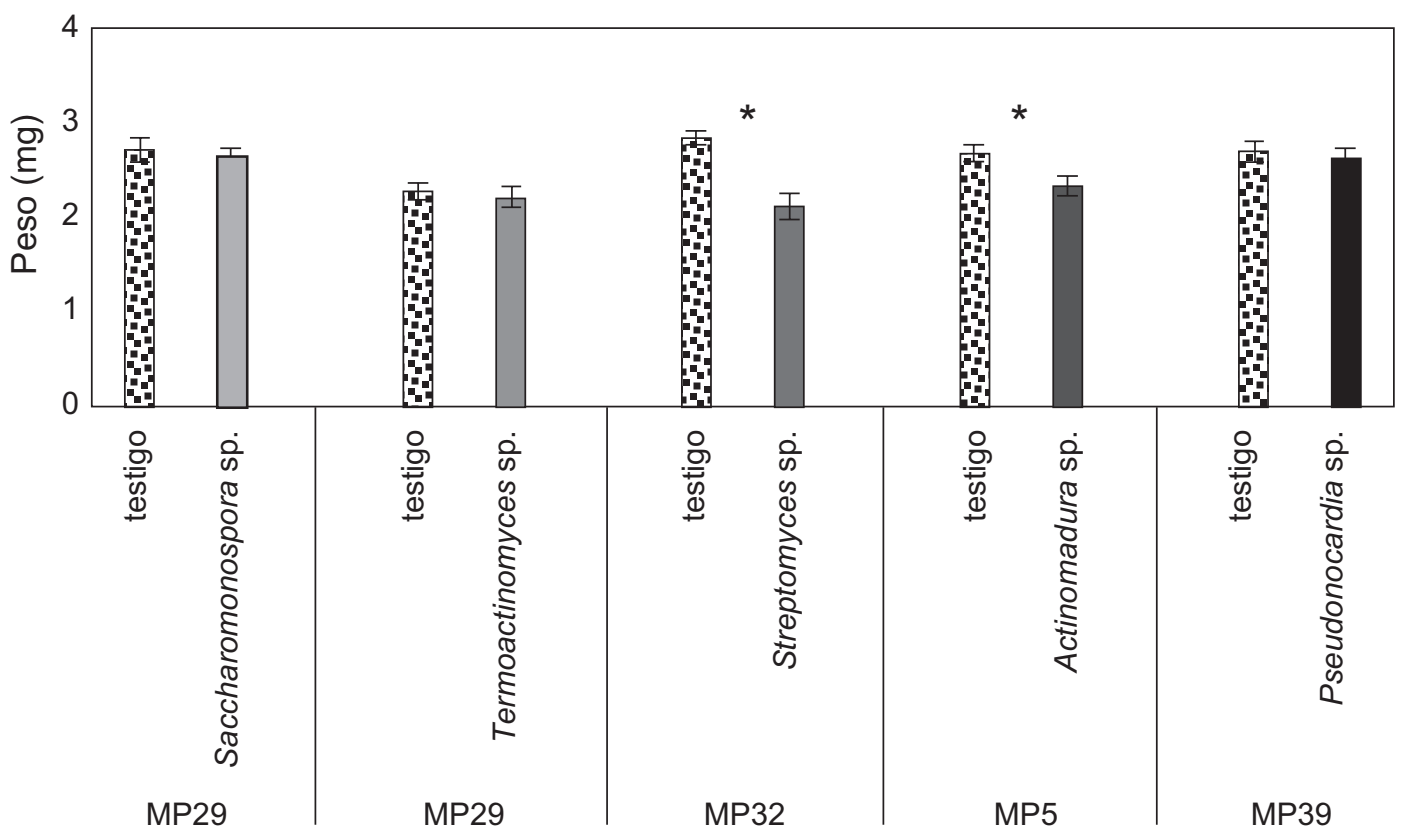

Fig. 2. Ensayo de degradación de PP en medio líquido con pérdida de peso gravimétrica después de 45 días. Las barras lisas representan la media de los pesos de las láminas de polipropileno luego de la incubación con las actinobacterias estudiadas \pm error estándar. Los testigos de cada tratamiento están representados por las barras punteadas. *Tratamiento con diferencia significativa $(\mathrm{p}<0.05)$.

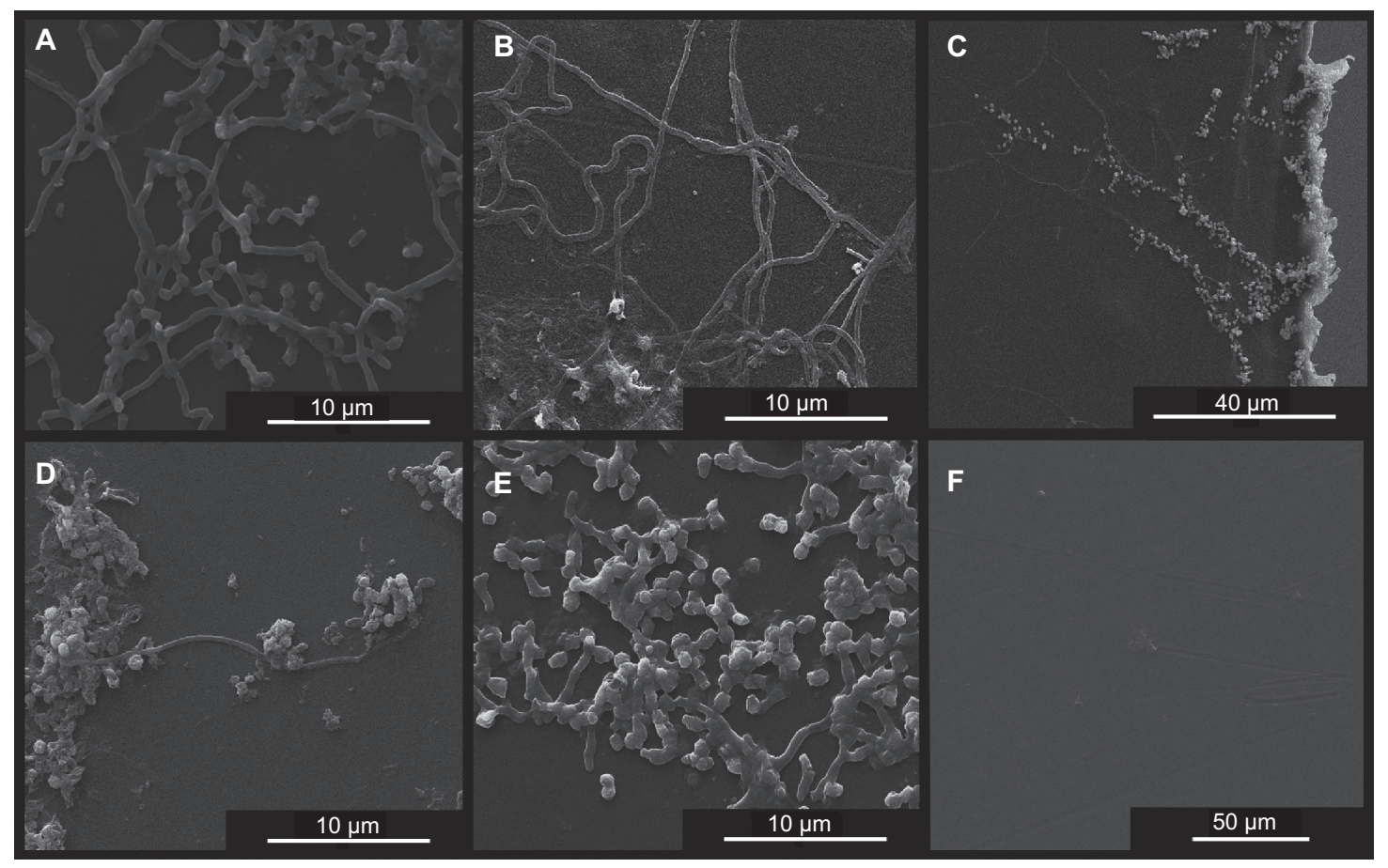

Fig. 3. Microfotografías de las distintas cepas de actinobacterias en crecimiento sobre la superficie del PP en los ensayos de degradación en medio líquido. (a) Actinomadura sp. MP5; (b) Streptomyces sp. MP32; (c) Saccharomonospora sp MP29; (d) Thermoactinomyces sp. MP22; (e) Pseudonocardia sp. MP39, y (f) testigo. 


\section{DISCUSIÓN}

El gran porcentaje de aislamientos del género Streptomyces obtenidos de las muestras de sustrato, coinciden con los datos bibliográficos, que indican que este género es el más abundante dentro de las actinobacterias del suelo (Korn-Wendisch y Kutzner 1992, McCarthy y Williams 1992).

El crecimiento a $50{ }^{\circ} \mathrm{C}$ indicaría que los aislamientos tienen un rango de temperatura de crecimiento termotolerante, pudiendo ser también termofílicos. Si bien en la descripción del género Streptomyces, se considera que la mayoría de estas bacterias son mesofílicas, también se cita la existencia de especies de este género que crecen en un rango termofílico (Kämpfer 2012). Las especies del género Saccharomonospora pueden tener un crecimiento en el rango mesófilo o termófilo, de 24 a $60{ }^{\circ} \mathrm{C}$ (Bum 2012). Las cepas de las especies termófilas de Saccharomonospora son normalmente aisladas de hábitats termófilos naturales tales como hojarasca, estiércol y composta, mientras que las especies mesófilas son frecuentemente encontradas en muestras de suelo (al-Zarban et al. 2002). Los microorganismos termófilos tienen una serie de características atractivas para la industria como altas tasas metabólicas y mayor estabilidad física y química de las enzimas en comparación con especies mesófilas metabólicamente similares. Esto les confiere una propiedad ventajosa para su aplicación industrial (Zeikus et al. 1981).

\section{Degradación de polímeros naturales}

Los resultados obtenidos en los ensayos de degradación de polímeros naturales apoyan el importante rol de las actinobacterias como recicladoras de material orgánico complejo. Esto se asocia con su capacidad de producir exoenzimas que descomponen sustancias de alto peso molecular a moléculas simples, las cuales pueden ingresar a la célula y ser metabolizadas. La capacidad de degradar almidón mediante exoenzimas es común en los microorganismos. Muchos hongos, bacterias y actinobacterias del suelo son productores activos de amilasas, las cuales hidrolizan el almidón soluble y/o gránulos de almidón a maltosa. Como estrategia para generar plásticos con mejores condiciones de degradación, se adiciona almidón a las cadenas poliméricas con el fin de facilitar la acción metabólica de los microorganismos y la biodegradación (Orhan y Büyükgüngör 2000). Las hemicelulosas son químicamente heterogéneas, pero relativamente susceptibles a la degradación, de tal manera que la actividad enzimática sobre el xilano (forma predominante de hemicelulosa) es muy común en actinobacterias (McCarthy y Williams 1992). La celulosa es atacada inicialmente por endoglucanasas, las cuales se liga al azar a las microfibrillas de celulosa en regiones amorfas; de esta manera producen hidrólisis en enlaces $\beta-1.4$ glucosídicos, generando múltiples sitios de ataque para las exo-1.4 $\beta$-Dglucanasa, las cuales actúan sobre los extremos no reductores de la cadena (Wilson 2011).

Esta actividad cooperativa es llamada sinergismo, ya que la suma de actividades de las enzimas combinadas es mayor que la suma de las actividades individuales para la degradación. El sinergismo endoglucanasa-exoglucanasa se explica considerando que la acción de las endoglucanasas crea nuevos sitios de acción para las exoglucanasas (Percival-Zhang et al. 2006, Wilson 2011). Debido a esta característica se presupone que, sin la acción de las endoglucanasas, es difícil que una exoglucanasa encuentre un sitio de acción. Esto concuerda con los resultados obtenidos en este trabajo, en el cual la mayoría de los aislamientos mostraron capacidad para producir principalmente endoglucanasas, aunque en menor medida algunas produjeron exoglucanasas. Los aislamientos con actividad exoglucanasa también produjeron actividad endoglucanasa. McCarthy y Williams (1992) exponen que la actividad endoglucanasa es común en actinobacterias, no así la actividad exoglucanasa, lo cual también se ve reflejado en los resultados obtenidos.

\section{Degradación de polipropileno en medio sólido}

El ensayo en medio sólido tiene como ventaja simular condiciones a las que podrían estar expuestos los polímeros sintéticos en el ambiente (exposición prolongada a radiaciones de alta energía, altas temperaturas, condiciones de sequía, entre otras). El PP es la forma metilada del PE y es considerado no biodegradable, debido a que cuenta con un alto nivel de hidrofobicidad y un alto peso molecular (Arráez et al. 2018, Hossain et al. 2018). Un medio de cultivo sin fuente de carbono, altas temperaturas y la acción de un solo microorganismo son condiciones poco óptimas para el desarrollo normal de las actividades metabólicas sobre este material. La biodegradación es un proceso muy lento, por lo que el tiempo es un factor importante. Futuros ensayos podrían rediseñarse ampliando el tiempo de cultivo para obtener mejores resultados, ya que probablemente en este trabajo el tiempo fue insuficiente para percibir el efecto de los microorganismos sobre este sustrato. Se han realizado estudios utilizando esta técnica en los cuales se observó biodegradación de láminas de polietileno con aditivos prooxidantes (Corti et al. 
2010). también probaron la biodegradabilidad de cuatro polímeros distintos, considerados biodegradables (ácido poliláctico, policaprolactona, mezcla almidón/policaprolactona y poli (butadién adipatoco-tereftalato). En ambos ensayos la naturaleza del material polimérico fue distinta a la estudiada en este trabajo, ya que lo polímeros utilizados poseen enlaces y grupos funcionales más susceptibles a la degradación en su configuración, lo que facilita el ataque por la acción de enzimas o resulta más sensible a la ruptura molecular por factores físicos como la temperatura o la radiación. Por ello estos resultados sientan bases de trabajo con PP como polímero no biodegradable en medio sólido. Si bien no se obtuvieron resultados significativos a nivel estadístico, se pudo observar crecimiento y colonización de la superficie por parte de las actinobacterias. Posiblemente el pretratamiento con luz UV produjo un grado de oxidación de las láminas de PP, lo que facilitó la colonización microbiana.

\section{Degradación de polipropileno en medio líquido}

A diferencia del cultivo en medio sólido, en los cultivos líquidos varían las condiciones, ya que el factor de agitación constante proporciona un medio homogéneo en cuanto a temperatura, humedad, oxígeno y nutrientes, facilitando el desarrollo de los microorganismos y colaborando con la colonización de la lámina de PP (la cual se encuentra totalmente sumergida en el medio) pretratada con luz UV. Estas diferencias pueden explicar por qué los mismos aislamientos sólo dieron resultados positivos respecto a la pérdida de peso del PP en el ensayo en medio líquido, sobre todo teniendo en cuenta que la velocidad de degradación depende de varios factores, entre ellos el área superficial del polímero, la actividad microbiana, el $\mathrm{pH}$, la temperatura y la presencia de nutrientes (Lee 1996).

Lee et al. (1991) realizaron ensayos de degradación en medio líquido en condiciones similares a las utilizadas en este trabajo con relación a temperatura de incubación, velocidad de agitación y pretratamiento con UV a $365 \mathrm{~nm}$; sin embargo, estos autores utilizaron material polimérico con prooxidantes y almidón adicionado a su estructura molecular y el tiempo de incubación fue de cuatro semanas. En este trabajo se aislaron tres actinobacterias del género Streptomyces (S. viridosporus T7A, S. badius 252 y $S$. setonii $75 \mathrm{Vi2}$ ) que mostraron degradación del sustrato. En un trabajo posterior, a partir de dichas cepas se preparó un cultivo con enzimas activas donde se colocaron las películas plásticas degradables y se incubaron con agitación durante tres semanas a $37^{\circ} \mathrm{C}$. Estos ensayos mostraron cambios en cuanto a las propiedades mecánicas y distribución del peso molecular del polietileno (Pometto et al. 1992). Otro trabajo en el que se utilizó medio líquido en similares condiciones mostró que cinco cepas de Streptomyces sp. exhibieron el mayor potencial para la degradación de PE (polímero no biodegradable), en comparación con hongos y otras bacterias como Pseudomonas sp., Bacillus sp., Staphylococcus sp. y Aspergillus sp. (Usha et al. 2011). En la India se probó la degradación de polietileno en medio líquido con actinobacterias aisladas de un manglar $\mathrm{y}$, coincidentemente con los trabajos anteriores, fueron dos cepas del género Streptomyces las que mostraron mayor capacidad de degradación del polímero respecto a las demás actinobacterias aisladas (Pseudonocardia, Actinoplanes, Sporichthya) (Sathya et al. 2012). Esto coincide con los resultados obtenidos en este trabajo al igual que con los trabajos de Sukkhum et al. (2009a, b), en los cuales se aisló una cepa termofílica del género Actinomadura con capacidad de degradación del ácido poli-L-láctico.

El objetivo de este trabajo fue determinar la capacidad de degradación de PP por las actinobacterias aisladas del vertedero de San Carlos de Bariloche. Los resultados preliminares son favorables en cuanto a la biodegradación de polímeros sintéticos por actinobacterias, representando un punto de partida para posibles desarrollos biotecnológicos.

\section{CONCLUSIÓN}

En el contexto de la amenaza ecológica que representa la acumulación de polímeros sintéticos en el ambiente, tanto la investigación como la búsqueda de nuevas herramientas biotecnológicas relacionadas con la biodegradación de estos materiales son de vital importancia. En este trabajo presentamos dos actinobacterias (Streptomyces sp. [MP32] y Actinomadura sp. [MP22]) como posibles degradadoras de PP parcialmente oxidado, uno de los polímeros sintéticos más estables y de mayor uso. De esta manera, y como punto de partida para futuras investigaciones, se puede considerar que las actinobacterias son una posible solución novedosa para tratar el problema de la contaminación por polímeros sintéticos. Por otro lado, la detección de cepas productoras de enzimas hidrolíticas extracelulares amplia el potencial biotecnológico de estas cepas, ya que su producción puede ser importante para su uso en la rama de alimentos, salud y remediación de la contaminación. 


\section{AGRADECIMIENTOS}

Agradecemos a la Lic. Agustina C. Cottet y a la Dra. Maria I. Messuti por sus aportes y comentarios para la preparación de este manuscrito, y a la operadora técnica P. Troyón (CM-GIA-GAATEN-CNEA). Este trabajo fue financiado por el Proyecto 04/B207 de la Secretaría de Investigación de la Universidad Nacional del Comahue y el Proyecto de Investigación PIP 0235-CONICET.

\section{REFERENCIAS}

Álvarez-Zeferino J.C., Cruz-Salas A.A., Vázquez-Morillas A. y Ojeda-Benítez S. (2020). Method for quantifying and characterization of microplastics in sand beaches. Rev. Int. Contam. Ambie. 36 (1), 151-164. https://doi. org/10.20937/RICA.2020.36.53540

Al-Zarban S.S., al-Musallam A.A., Abbas I., Stackebrandt E. y Kroppenstedt R.M. (2002). Saccharomonospora halophila sp. nov., a novel halophilic actinomycete isolated from marsh soil in Kuwait. Int. J. Syst. Evol. Microbiol. 52 (2), 555-558. https://doi. org/10.1099/00207713-52-2-555

Amoroso M.J., Benimeli C.S. y Cuozzo S.A. (2013). Actinobacteria: Application in bioremediation and production of industrial enzymes. CRC Press, Boca Ratón, EUA, 298 pp.

Arráez F.J., Arnal M.L. y Müller A.J. (2018). Thermal and UV degradation of polypropylene with pro-oxidant. Abiotic characterization. J. Appl. Polym. Sci. 135 (14), 46088. https://doi.org/10.1002/app.46088

Balasubramanian V., Natarajan K., Hemambika B., Ramesh N., Sumathi, C.S., Kottaimuthu R. y Rajesh Kannan V. (2010). High density polyethylene (HDPE) degrading potential bacteria from marine ecosystem of Gulf of Mannar, India. Lett. Appl. Microbiol. 51 (2), 205-211. https://doi.org/10.1111/j.1472-765X.2010.02883.x

Bowen G.D. y Rovira A.D. (1999). The rhizosphere and its management to improve plant growth. Adv. Agron. 66, 1-102. https://doi.org/10.1016/s0065-2113(08)60425-3

Bühler M.D., de Torres Curth M. y Garibaldi L.A. (2013). Demography and socioeconomic vulnerability influence fire occurrence in Bariloche (Argentina). Landscape Urban Plan. 110, 64-73. https://doi. org/10.1016/j.landurbplan.2012.10.006

Bum Kim S. (2012). Genus XIII. Saccharomonospora Nonomura and Ohara 1971, 899 ${ }^{\mathrm{AL}}$. En: Bergey's manual of systematic bacteriology (Goodfellow M., Kämpfer P., Busse H.J., Trujillo M., Suzuki K.I., Ludwig W. y Whitman W.B., Eds.). Springer, Nueva York, EUA, 1390-1396.
Corti A., Muniyasamy S., Vitali M., Imam S.H. y Chiellini E. (2010). Oxidation and biodegradation of polyethylene films containing pro-oxidant additives: Synergistic effects of sunlight exposure, thermal aging and fungal biodegradation. Polym. Degrad. Stabil. 95 (6), 1106-1114. https://doi.org/10.1016/j.polymdegradstab.2010.02.018

Cross T. (1989). The Actinomycetes II: Growth and examination of Actinomycetes - some guidelines. En: Bergey's manual of systematic bacteriology (Williams S.T., Sharpe M.E. y Holt J.C., Eds.). Williams y Wilkins, Baltimore, EUA, 2340-2343.

Dworkin M., Falkow S., Rosenberg E., Schleifer K.H. y Stackebrandt E. (2007). The prokaryotes: a handbook on the biology of bacteria. Archaea. Bacteria: Firmicutes. Actinomycetes. Springer, Nueva York, EUA, $1144 \mathrm{pp}$.

Emerson R. (1958). Mycological organization. Mycologia 50, 589- 621. https://doi.org/10.1080/00275514.1958 .12024755

Goodfellow M. (2012). Phylum XXVI. Actinobacteria phyl. nov. En: Bergey's manual of systematic bacteriology (Goodfellow M., Kämpfer P., Busse H.J., Trujillo M., Suzuki K.I., Ludwig W. y Whitman W.B., Eds.). Springer, Nueva York, EUA, 1-28.

Hossain M.R., Jiang M., Wei Q. y Leff L.G. (2018). Microplastic surface properties affect bacterial colonization in freshwater. J. Basic Microbial. 59 (1), 54-61. https:// doi.org/10.1002/jobm.201800174

Kasana C.R., Salwan R., Dhar H., Dutt S. y Gulati A. (2008). A rapid and easy method for the detection of microbial cellulases on agar plates using gram's iodine. Curr. Microbiol. 57, 503-507. https://doi.org/10.1007/ s00284-008-9276-8

Kämpfer P. (2012). Order XIV. Streptomycetales ord. nov. En: Bergey's manual of systematic bacteriology (Goodfellow M., Kämpfer P., Busse H.J., Trujillo M., Suzuki K.I., Ludwig W. y Whitman W.B., Eds.). Springer, Nueva York, EUA, 1446-1806.

Korn-Wendisch F. y Kutzner H. (1992). The family Streptomycetaceae. En: The Prokaryotes, a handbook on the biology of bacteria, ecophysiology, isolation, application (Ballows A., Trüper H.G., Dworkin M., Harder W. y Schleifer K.H., Eds.). Springer, Nueva York, EUA, 921-995.

Krueger M.C., Harms H. y Schlosser D. (2015). Prospects for microbiological solutions to environmental pollution with plastics. Appl. Microbiol. Biotech. 99 (21), 8857-8874. https://doi.org/10.1007/s00253-0156879-4

Lee B., Pometto A.L., Fratzke A. y Bailey T.B. (1991). Biodegradation of degradable plastic polyethylene by Phanerochaete and Streptomyces species. Appl. 
Environ. Microbiol. 57 (3), 678-685. https://doi. org/10.1128/aem.57.3.678-685.1991

Lee S.Y. (1996). Bacterial polyhydroxyalkanoates. Biotechnol. Bioeng. 49, 1-14. https://doi.org/10.1002/ (SICI) 1097-0290(19960105)49:1<1::AIDBIT1>3.0.CO;2-P.

Massardier-Nageotte V., Pestre C., Cruard-Pradet T. y Bayard R. (2006). Aerobic andanaerobic biodegradability of polymer films and physico-chemical characterization. Polym. Degrad. Stab. 91, 620-627. https://doi. org/10.1016/j.polymdegradstab.2005.02.029

Mergaert J. y Swings J. (1996). Biodiversity of microorganisms that degrade bacterial and synthetic polyesters. J. Ind. Microbiol. Biot. 17, 463-469. https://doi. org/10.1007/BF01574777

Mason S.A., Garneau D., Sutton R. y Chu Y. (2016). Microplastic pollution is widely detected in US municipal wastewater treatment plan effluent. Environ. Pollut. 218, 1045-54. https://doi.org/10.1016/j. envpol.2016.08.056

McCarthy A.J. y Williams S.T. (1992). Actinomycetes as agents of biodegradation in the environment - A review. Gene 115, 189-192. https://doi. org/10.1016/0378-1119(92)90558-7

Miyadoh S., Hamada M., Hotta K., Kudo T., Seino A., Vobis G. y Yokota A. (1997). Atlas of Actinomycetes. The Society for Actinomycetes Japan, Asakura, Tokyo, 223 pp.

Mor R. y Sivan A. (2008). Biofilm formation and partial biodegradation of polystyrene by the actinomycete Rhodococcus ruber. Biodegradation 19, 851-858. https://doi.org/10.1007/s10532-008-9188-0

Oberbeckmann S., Löder M.G. y Labrenz M. (2015). Marine microplastic associated biofilms - A review. Environ. Chem. 12, 551-62. https://doi.org/10.1071/ en15069

Ojha N., Pradhan N., Singh S., Barla A., Shrivastava A., Khatua P., Rai V. y Bose S. (2017). Evaluation of HDPE and LDPE degradation by fungus, implemented by statistical optimization. Sci. Rep. 7 (1), 1-13. https:// doi.org/10.1038/srep39515

Orhan Y. y Büyükgüngor H. (2000). Enhancement of biodegradability of disposable polyethylene in controlled biological soil. Int. Biodeter. Biodegr. 45, 49-55. https://doi.org/10.1016/S0964-8305(00)00048-2

Percival-Zhang Y.-H., Himmel M.E. y Mielenz J.R. (2006). Outlook for cellulase improvement: Screening and selection strategies. Biotechnol. Adv. 24 (5), 452481. https://doi.org/10.1016/j.biotechadv.2006.03.003

Pometto A.L., Lee B. y Johnson K.E. (1992). Production of an extracellular polyethylene-degrading enzyme(s) by Streptomyces sp. Appl. Environ. Microbiol. 58, 731733. https://doi.org/10.1128/aem.58.2.731-733.1992
Restrepo-Flórez J.M., Bassi A. y Thompson M.R. (2014). Microbial degradation and deterioration of polyethylene - A review. Int. Biodeter. Biodegr. 88, 83-90. https://doi.org/10.1016/j.ibiod.2013.12.014

Rivas R., Sánchez M., Trujillo M.E., Zurdo-Piñeiro J.L., Mateos P.F., Martínez-Molina E. y Velázquez E. (2003). Xylanimonas cellulosilytica gen. nov., sp. nov., a xylanolytic bacterium isolated from a decayed tree (Ulmus nigra). Int. J. Syst. Evol. Microbiol. 53 (1), 99-103. https://doi.org/10.1099/ijs.0.02207-0

Rohban R., Amoozegar M.A. y Ventosa A. (2008). Screening and isolation of halophilic bacteria producing extracellular hydrolyses from Howz Soltan Lake, Iran. J. Ind. Microbiol. Biotechnol. 36 (3), 333-340. https:// doi.org/10.1007/s10295-008-0500-0

Sathya R., Ushadevi T. y Panneerselvam A. (2012). Plastic degrading actinomycetes isolated from mangrove sediments. Int. J. Curr. Res. 4 (10), 001-003.

Shimao M. (2001). Biodegradation of plastics. Curr. Opin. Biotech. 12, 242-247. https://doi.org/10.1016/S09581669(00)00206-8

Shirling E.B. y Gottlieb D. (1966). Methods for characterization of Streptomyces species. Int. J. Syst. Bacteriol. 16, 313-340. https://doi.org/10.1099/00207713-163-313

Singh B. y Sharma N. (2008). Mechanistic implications of plastic degradation. Polym. Degrad. Stab. 93 (3), 561-584. https://doi.org/10.1016/j.polymdegradstab.2007.11.008

Sivan A., Szanto M. y Pavlov V. (2006). Biofilm development of the polyethylene- degrading bacterium Rhodococcus ruber. Appl. Microbiol. Biotec. 72, 346-352. https://doi.org/10.1007/s00253-005-0259-4

Sukkhum S., Tokuyama S., Tamura, T. y Kitpreechavanich V. (2009a). A novel poly (L-lactide) degrading actinomycetes isolated from Thai forest soil, phylogenic relationship and the enzyme characterization. J. Gen. Appl. Microbiol. 55 (6), 459-467. https://doi. org/10.2323/jgam.55.459

Sukkhum S., Tokuyama S. y Kitpreechavanich V. (2009b) Development of fermentation process for PLA-degrading enzyme production by a new thermophilic Actinomadura sp. T16-1. Biotechnol. Bioproc. 14, 302-306. https://doi.org/10.1007/ s12257-008-0207-0

Usha R., Sangeetha T. y Palaniswamy M. (2011). Screening of polyethylene degrading microorganisms from garbage soil. Libyan Agric. Res. Cen. J. Int. 2 (4), 200-204.

Vobis G. y Chaia E. (1998). El rol ecológico de los actinomycetes en el suelo. En: Actas del XVI Congreso Argentino de la Ciencia del Suelo (AACS), Villa Carlos Paz, Córdoba, Argentina, 375-381. 
Vobis G. (2006). The genus Actinoplanes and related genera. En: The Prokaryotes, a handbook on the biology of bacteria. 3a. ed. Vol. 3: Archaea and Bacteria: Firmicutes, Actinomycetes (Dworkin M., Falkow S., Rosenberg E., Schleifer K.-H. y Stackebrandt E., Eds.). Springer, Nueva York, 623-653. https://doi. org/10.1007/0-387-30743-5_24
Wilson D.B. (2011). Microbial diversity of cellulose hydrolysis. Curr. Op. Microbiol. 14 (3), 259-263. https:// doi.org/10.1016/j.mib.2011.04.004

Zeikus J.G., Ben-Bassat A., Ng T.K. y Lamed R.J. (1981). Thermophilic ethanol fermentations. En: Trends in the biology of fermentations for fuels and chemicals. Springer, Boston, EUA, 441-461. https://doi. org/10.1007/978-1-4684-3980-9_26 